Equivalence between Kaluza Klein modes of gravitinos and goldstinos in brane induced supersymmetry breaking

This content has been downloaded from IOPscience. Please scroll down to see the full text.

JHEP01(2004)052

(http://iopscience.iop.org/1126-6708/2004/01/052)

View the table of contents for this issue, or go to the journal homepage for more

Download details:

IP Address: 147.96.14.16

This content was downloaded on 25/09/2015 at $12: 53$

Please note that terms and conditions apply. 


\title{
Equivalence between Kaluza Klein modes of gravitinos and goldstinos in brane induced supersymmetry breaking
}

\author{
Stefania De Curtis \\ I.N.F.N., Sezione di Firenze, I-50019 Sesto F., Italy \\ E-mail: decurtis@fi.infn.it

\section{Daniele Dominici} \\ Dipartimento di Fisica, Università di Firenze \\ I-50019 Sesto F., Italy, and \\ I.N.F.N., Sezione di Firenze, I-50019 Sesto F., Italy \\ E-mail: dominici@fi.infn.it
}

\section{José R. Pelaez}

Dept. de Física Teórica II, Univ. Complutense

28040 Madrid, Spain

E-mail: jrpelaez@fis.ucm.es

ABSTRACT: We identify the goldstino fields that give mass to the Kaluza Klein modes of five dimensional supergravity, when supersymmetry breaking is induced by brane effects. We then proof the four dimensional Equivalence Theorem that, in renormalizable gauges, allows for the replacement of Kaluza Klein modes of helicity $\pm 1 / 2$ gravitinos in terms of goldstinos. Finally we identify the five dimensional renormalizable gauge fixing that leads to the Equivalence Theorem.

Keywords: Field Theories in Higher Dimensions, Supergravity Models, 


\section{Contents}

1. Introduction 1

2. The bulk and brane actions 2

3. Mass matrix eigenstates 5

4. The Equivalence Theorem 7

$4.1 R_{\xi}$ gauge fixing 8

4.2 The Equivalence Theorem for KK modes

4.3 The $R \rightarrow 0$ limit 10

5. Conclusions 10

A. Notation 11

B. Gauge fixing term in 5D supergravity 11

\section{Introduction}

Extra dimensional models provide a geometrical approach to tackle the hierarchy problem in particle physics. In addition, they are suggested by recent developments of string theory. In many of these models supersymmetry plays a relevant role in generating or stabilizing the hierarchies. This is for instance the case of the M-theory extensions of the heterotic $E_{8} \times E_{8}$ string, which lead to a scenario with two branes at the end of a finite dimension 国. The bulk is populated by supergravity whereas matter and gauge fields are constrained to the branes. For our purposes, we will neglect the six dimensions compactified on the Calabi-Yau manifold and we will consider an effective five dimensional (5D) supergravity with two four dimensional (4D) walls containing the matter and the gauge fields. In these models the supersymmetry breaking can be induced by brane localized dynamics [2, (5, : 1 leading to massive gravitinos through the super Higgs mechanism.

From the four dimensional point of view, however, the gravitino becomes a tower of massive Kaluza Klein (KK) gravitinos, whose mass is due to the brane supersymmetry breaking effects and the five dimensional compactification. Actually, the gravitino mass eigenfields are a complicated combination of the Fourier modes of the 5D gravitino fields. The zero KK mode was identified in [3] and the whole tower in [4. Intuitively, gravitinos become massive because they acquire helicity $\pm 1 / 2$ components by eating the goldstino degrees of freedom. Indeed there is an Equivalence Theorem (ET) for 4D supergravity, that 
relates at high energy the scattering amplitudes of helicity $\pm 1 / 2$ gravitinos to amplitudes where these gravitinos are replaced by their associated goldstinos [5]. This theorem is very useful in calculating gravitino observables since it is much easier to handle spin $1 / 2$ particles, as goldstinos, than complicated helicity projections of Rarita-Schwinger spin $3 / 2$ fields. One of the most typical applications is the use of the ET to calculate the cosmological production and decay of massive gravitinos [6], that impose severe constraints in the parameter space of supergravity and/or cosmology if standard nucleosynthesis is to be preserved. It has been recently pointed out that the gravitino Kaluza Klein modes are likely to pose a bigger challenge to nucleosynthesis [7], but the calculation of their abundance was postponed until the formal approach for their study was available. In this work we provide such a framework. Another common application of the ET is the study of perturbative unitarity limits, since it is well known that tree level amplitudes containing helicity $\pm 1 / 2$ gravitinos can violate tree level unitarity [8. In this respect, it has been recently shown that the introduction of extra dimensions could worsen dramatically the problem of unitarity unless the interactions are conveniently suppressed to balance the increase of KK states with the available energy [9]. In principle, such effects also occur for $5 \mathrm{D}$ gravitinos.

The zero mode goldstino for the effective 5D supergravity was already identified in [3, 4. However, the formulation of the ET in extra dimensional models presents the additional complication that goldstinos are also a mixture of KK components that depends nontrivially on both the brane and the bulk dynamics [10]. Also, the ET is formulated in the so called renormalizable t'Hooft-Veltman, or $R_{\xi}$ gauges, while these kind of models are

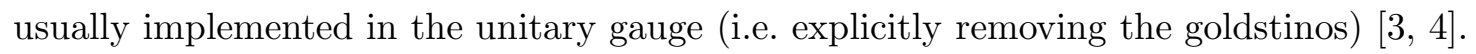

In this work, we present a complete proof of the ET in brane induced supersymmetry breaking scenarios, relating each KK mode of the gravitinos with their corresponding combination of KK goldstinos. In section 2 we introduce the details of the model. In section 3 we perform the diagonalization of the gravitino and goldstino mass terms as well as their mixing term. In section 1 we prove the ET, by identifying first the gravitino and goldstino mass eigenfields and the four dimensional $R_{\xi}$ gauge fixing. At the end of the section we present the gauge fixing in its five dimensional form, and we study the limit of small extra dimensions where the ET is particularly simple even in terms of the initial fields. In section 5 we summarize.

\section{The bulk and brane actions}

The action that reproduces all the main features of gaugino condensation [2 in M-theory [1] at the level of the five dimensional effective theory is given in 四. The fifth dimension is compactified on the $S^{1} / Z_{2}$ orbifold, obtained by identifying $x^{5} \leftrightarrow-x^{5}$. Customarily $x^{5}$ varies in the interval $[0, \pi \kappa]$. Introducing a $1 / 2$ factor to avoid overcounting, the bulk action is defined to be:

$$
S_{\text {bulk }}=\frac{1}{2} \int d^{4} x \int_{-\pi \kappa}^{+\pi \kappa} d x^{5} \mathcal{L}_{\text {bulk }}
$$


where $\mathcal{L}_{\text {bulk }}$ is the $5 \mathrm{D}$ supergravity lagrangian density [11]. The five dimensional coordinates are $x^{M}=\left(x^{m}, x^{5}\right)$ and the fields are fluctuations over the following background:

$$
\left\langle g_{M N}\right\rangle=\left(\begin{array}{cc}
\eta_{m n} & 0 \\
0 & r^{2}
\end{array}\right)
$$

where $R=r / M_{5}$ is the physical compactification radius, $M_{5}=\kappa^{-1}$ being the $5 \mathrm{D}$ (reduced) Planck mass related to the $4 \mathrm{D}$ Planck mass by $M_{4}^{2}=\pi R M_{5}^{3}$. Two gravitino fields are needed to form a generalized Majorana 5D spinor, in particular, for the gravity multiplets, we have: $\Psi=\left(\psi_{\alpha}^{1}, \bar{\psi}^{2} \dot{\alpha}\right)$ and $\bar{\Psi}=\left(\psi^{2 \alpha}, \bar{\psi}_{\dot{\alpha}}\right)$. We assign even $Z_{2}$-parity to $e_{m}^{a}, e_{5 \hat{5}}, \psi_{m}^{1}, \psi_{5}^{2}$ and odd $Z_{2}$-parity to $e_{5}^{a}, e_{m \hat{5}}, \psi_{m}^{2}, \psi_{5}^{1}$, here $e_{M}^{A}$ is the fünfbein and $\hat{5}$ is the fifth tangentspace index. We will denote by $e_{4}\left(e_{5}\right)$ the determinant of the vierbein (fünfbein), although, since we will be interested in fermion bilinears only, we will set it to 1 for simplicity in most cases. From the $4 \mathrm{D}$ point of view, the zero modes of $e_{m}^{a}$ and $\psi_{m}^{1}$, with spins $(2,3 / 2)$ respectively, form a massless $N=1$ gravitational supermultiplet. The fields $e_{5 \hat{5}}$ and $\psi_{5}^{2}$ form, together with the $B_{5}$ component of the graviphoton $B_{M}$, a $N=1$ massless chiral multiplet. In addition one has an infinite Kaluza Klein (KK) series of multiplets of $\mathrm{N}=2$ supergravity with spins $(2,3 / 2,3 / 2,1)$ and masses $M_{n}^{2}=n^{2} / R^{2}, n=1,2 \ldots$. As we will see in detail below, the KK gravitino tower gets mass through an infinite series of super Higgs effects by eating the KK modes of the goldstino field.

Brane physics is not relevant for our purposes. We will follow 14 and imagine that the branes are located at orbifold fixed points without tension-like terms and without generating a warp factor so that the bosonic vacuum is flat. We also assume that fields living in the brane are integrated out leaving a constant superpotential vacuum expectation value (vev) on each brane. It has been shown in 畞 that these vev's can spontaneously break the remaining $N=1$ supersymmetry. Since the physics can be different on both branes the vev's are parametrized by two constants $P_{0}$ and $P_{\pi}$ (which we have taken real for simplicity) with dimension mass $^{3}$. Thus [2, 3, 国:

$$
S_{\text {brane }}=\frac{\kappa^{2}}{2} \int d^{4} x \int_{-\pi \kappa}^{\pi \kappa} d x^{5}\left[\delta\left(x^{5}\right) P_{0}+\delta\left(x^{5}-\pi \kappa\right) P_{\pi}\right] \psi_{m}^{1} \sigma^{m n} \psi_{n}^{1}+\text { h.c. }
$$

In order to study the super Higgs effect and the equivalence between KK goldstinos and gravitinos, it is enough to consider the $5 \mathrm{D}$ fermionic bilinear terms:

$$
\begin{aligned}
S_{2 f}^{(5)}= & \frac{1}{2} \int d^{4} x \int_{-\pi \kappa}^{+\pi \kappa} d x^{5} \times \\
\times & \left\{\frac{r}{\kappa} \epsilon^{m n p q}\left(\bar{\psi}_{m}^{1} \bar{\sigma}_{n} \partial_{p} \psi_{q}^{1}-\psi_{m}^{2} \sigma_{n} \partial_{p} \bar{\psi}_{q}^{2}\right)+\right. \\
& +\frac{2}{\kappa} e_{4}\left(-\psi_{m}^{2} \sigma^{m n} \partial_{5} \psi_{n}^{1}+\bar{\psi}_{m}^{1} \bar{\sigma}^{m n} \partial_{5} \bar{\psi}_{n}^{2}+\psi_{m}^{2} \sigma^{m n} \partial_{n} \psi_{5}^{1}-\right. \\
& \left.\quad-\bar{\psi}_{m}^{1} \bar{\sigma}^{m n} \partial_{n} \bar{\psi}_{5}+\psi_{5}^{2} \sigma^{m n} \partial_{m} \psi_{n}^{1}-\bar{\psi}_{5}^{1} \bar{\sigma}^{m n} \partial_{m} \bar{\psi}_{n}^{2}\right)+ \\
& \left.+\left(e_{4} \kappa^{2}\left[\delta\left(x^{5}\right) P_{0}+\delta\left(x^{5}-\pi \kappa\right) P_{\pi}\right] \psi_{m}^{1} \sigma^{m n} \psi_{n}^{1}+\text { h.c. }\right)\right\} .
\end{aligned}
$$


In contrast to [4], where the $\Psi^{5}$ field is eliminated by going to the unitary gauge, we are interested in identifying the goldstinos eaten by the gravitinos to acquire their mass through the Higgs mechanism. For that reason it is convenient to perform several transformations that will allow us to diagonalize simultaneously the gravitino and goldstino mass matrices as well as their mixing term. Let us follow [12] and transform the fields

$$
\Psi_{m} \rightarrow \Psi_{m}+\frac{1}{\sqrt{6}} \Gamma_{m} \Gamma^{5} \Psi_{5}, \quad \Psi_{5} \rightarrow \frac{2 r}{\sqrt{6}} \Psi_{5}
$$

(an equivalent transformation, but with slightly different redefinitions, can be found also in [3]). We obtain:

$$
\begin{aligned}
S_{2 f}^{(5)}=\frac{1}{2} \int d^{4} x \int_{-\pi \kappa}^{+\pi \kappa} d x^{5}\{ & \frac{r}{\kappa} \epsilon^{m n p q}\left(\bar{\psi}_{m}^{1} \bar{\sigma}_{n} \partial_{p} \psi_{q}^{1}-\psi_{m}^{2} \sigma_{n} \partial_{p} \bar{\psi}_{q}^{2}\right)- \\
& -i \frac{r e_{4}}{\kappa}\left(\psi_{5}^{2} \sigma^{m} \partial_{m} \bar{\psi}^{2}{ }_{5}+\bar{\psi}^{1} \bar{\sigma}^{m} \partial_{m} \psi_{5}^{1}\right)+ \\
& +\frac{2 e_{4}}{\kappa}\left(\bar{\psi}_{5}^{1} \partial_{5} \bar{\psi}^{2}{ }_{5}-\psi_{5}^{2} \partial_{5} \psi_{5}^{1}\right)+ \\
& +\frac{i \sqrt{6} e_{4}}{2 \kappa}\left(\bar{\psi}^{1} \bar{\sigma}^{m} \partial_{5} \psi_{m}^{1}+\psi_{m}^{2} \sigma^{m} \partial_{5} \bar{\psi}_{5}^{2}+\text { h.c. }\right)+ \\
& +\frac{2 e_{4}}{\kappa}\left(-\psi_{m}^{2} \sigma^{m n} \partial_{5} \psi_{n}^{1}+\bar{\psi}_{m}^{1} \bar{\sigma}^{m n} \partial_{5} \bar{\psi}_{n}^{2}\right)+ \\
& \left.+\left(e_{4} \kappa^{2}\left[\delta\left(x^{5}\right) P_{0}+\delta\left(x^{5}-\pi \kappa\right) P_{\pi}\right] \psi_{m}^{1} \sigma^{m n} \psi_{n}^{1}+\text { h.c. }\right)\right\}+ \\
+\left(e _ { 4 } \kappa ^ { 2 } \left[\delta\left(x^{5}\right) P_{0}+\right.\right. & \left.\left.\left.\delta\left(x^{5}-\pi \kappa\right) P_{\pi}\right]\left[\frac{-i \sqrt{6}}{2}\left(\psi_{m}^{1} \sigma^{m} \bar{\psi}_{5}^{2}\right)+\bar{\psi}_{5}^{2} \bar{\psi}_{5}^{2}\right]+\text { h.c. }\right)\right\} .
\end{aligned}
$$

Note that, in this way, we have generated a standard kinetic term for the $\Psi_{5}$ field that was not present before. We will need this term to prove the equivalence between helicity $\pm 1 / 2$ gravitinos and goldstinos. We can now write the $4 \mathrm{D}$ reduction of the above lagrangian, recalling that the even fields $\psi^{\text {even }}=\psi_{m}^{1}, \psi_{5}^{2}$ have the following Fourier expansion:

$$
\psi^{\text {even }}\left(x^{5}\right)=\frac{1}{\sqrt{\pi r}}\left[\psi_{0}^{\text {even }}+\sqrt{2} \sum_{\rho=1}^{\infty} \psi_{\rho}^{\text {even }} \cos \left(\rho M_{5} x^{5}\right)\right]
$$

whereas the odd fields $\psi^{\text {odd }}=\psi_{m}^{2}, \psi_{5}^{1}$ have:

$$
\psi^{\text {odd }}\left(x^{5}\right)=\frac{\sqrt{2}}{\sqrt{\pi r}} \sum_{\rho=1}^{\infty} \psi_{\rho}^{\text {odd }} \sin \left(\rho M_{5} x^{5}\right),
$$

consistently with their $Z_{2}$-parity assignments. After integration of the $x^{5}$ coordinate, we find the following $4 \mathrm{D}$ lagrangian (we consider only the part of the lagrangian which is quadratic in the fermion fields):

$$
\mathcal{L}_{2 f}^{(4)}=\left\{\frac{1}{2} \epsilon^{m n p q}\left({\overline{\psi^{1}}}_{p, 0} \bar{\sigma}_{q} \partial_{m} \psi_{n, 0}^{1}+\sum_{\rho=1}^{\infty} \bar{\psi}_{p, \rho}^{1} \bar{\sigma}_{q} \partial_{m} \psi_{n, \rho}^{1}+\sum_{\rho=1}^{\infty}{\overline{\psi^{2}}}_{p, \rho} \bar{\sigma}_{q} \partial_{m} \psi_{n, \rho}^{2}\right)-\right.
$$




$$
\begin{aligned}
& -\frac{i}{2}\left({\overline{\psi^{2}}}_{5,0} \bar{\sigma}^{m} \partial_{m} \psi_{5,0}^{2}+\sum_{\rho=1}^{\infty}{\overline{\psi^{2}}}_{5, \rho} \bar{\sigma}^{m} \partial_{m} \psi_{5, \rho}^{2}+\sum_{\rho=1}^{\infty} \bar{\psi}^{1}{ }_{5, \rho} \bar{\sigma}^{m} \partial_{m} \psi_{5, \rho}^{1}\right)+ \\
& +\frac{2}{r} \sum_{\rho=1}^{\infty}\left(\rho M_{5}\right)\left(\psi_{m, \rho}^{2} \sigma^{m n} \psi_{n, \rho}^{1}-\psi_{5, \rho}^{1} \psi_{5, \rho}^{2}\right)- \\
& -\frac{i \sqrt{6}}{2 r} \sum_{\rho=1}^{\infty}\left(\rho M_{5}\right)\left(\bar{\psi}_{5, \rho} \bar{\sigma}^{m} \psi_{m, \rho}^{1}+\psi_{5, \rho}^{2} \sigma^{m} \bar{\psi}_{5, \rho}\right)+ \\
& +\frac{\kappa^{2}}{2 \pi r} P_{0}\left[\left(\psi_{m, 0}^{1}+\sqrt{2} \sum_{\rho=1}^{\infty} \psi_{m, \rho}^{1}\right) \sigma^{m n}\left(\psi_{n, 0}^{1}+\sqrt{2} \sum_{\sigma=1}^{\infty} \psi_{n, \sigma}^{1}\right)+\right. \\
& \left.+\left({\overline{\psi^{2}}}_{5,0}+\sqrt{2} \sum_{\rho=1}^{\infty}{\overline{\psi^{2}}}_{5, \rho}\right)\left({\overline{\psi^{2}}}_{5,0}+\sqrt{2} \sum_{\sigma=1}^{\infty}{\overline{\psi^{2}}}_{5, \sigma}\right)\right]+ \\
& +\frac{\kappa^{2}}{2 \pi r}\left[P_{\pi}\left(\psi_{m, 0}^{1}+\sqrt{2} \sum_{\rho=1}^{\infty}(-1)^{\rho} \psi_{m, \rho}^{1}\right) \sigma^{m n}\left(\psi_{n, 0}^{1}+\sqrt{2} \sum_{\sigma=1}^{\infty}(-1)^{\sigma} \psi_{n, \sigma}^{1}\right)+\right. \\
& \left.+\left({\overline{\psi^{2}}}_{5,0}+\sqrt{2} \sum_{\rho=1}^{\infty}(-1)^{\rho}{\overline{\psi^{2}}}_{5, \rho}\right)\left({\overline{\psi^{2}}}_{5,0}+\sqrt{2} \sum_{\sigma=1}^{\infty}(-1)^{\sigma}{\overline{\psi^{2}}}_{5, \sigma}\right)\right]- \\
& -i \frac{\sqrt{6} \kappa^{2}}{4 \pi r} P_{0}\left(\psi_{m, 0}^{1}+\sqrt{2} \sum_{\rho=1}^{\infty} \psi_{m, \rho}^{1}\right) \sigma^{m}\left(\bar{\psi}_{5,0}^{1}+\sqrt{2} \sum_{\sigma=1}^{\infty} \bar{\psi}_{5, \sigma}^{2}\right)- \\
& \left.-i \frac{\sqrt{6} \kappa^{2}}{4 \pi r} P_{\pi}\left(\psi_{m, 0}^{1}+\sqrt{2} \sum_{\rho=1}^{\infty}(-1)^{\rho} \psi_{m, \rho}^{1}\right) \sigma^{m}\left({\overline{\psi^{2}}}_{5,0}+\sqrt{2} \sum_{\sigma=1}^{\infty}(-1)^{\sigma}{\overline{\psi^{2}}}_{5, \sigma}\right)\right\}+ \text { h.c. }
\end{aligned}
$$

\section{Mass matrix eigenstates}

The gravitino mass matrix has been shown to diagonalize under the following transformations. First, by defining [4]

$$
\psi_{m, \rho}^{ \pm}=\frac{\psi_{m, \rho}^{1} \pm \psi_{m, \rho}^{2}}{\sqrt{2}}, \quad(\rho>0), \quad P_{ \pm}=\frac{\kappa^{3}}{2 \pi}\left(P_{0} \pm P_{\pi}\right),
$$

in the basis $\mathbf{\Psi}_{m}=\left(\psi_{m, 0}^{1}, \psi_{m, 1}^{+}, \psi_{m, 1}^{-}, \psi_{m, 2}^{+}, \psi_{m, 2}^{-}, \ldots\right)^{T}$ the mass matrix reads:

$$
\mathcal{M}_{3 / 2}=\frac{1}{R}\left(\begin{array}{c|cc|cc|c}
P_{+} & P_{-} & P_{-} & P_{+} & P_{+} & \cdots \\
\hline P_{-} & P_{+}+1 & P_{+} & P_{-} & P_{-} & \cdots \\
P_{-} & P_{+} & P_{+}-1 & P_{-} & P_{-} & \cdots \\
\hline P_{+} & P_{-} & P_{-} & P_{+}+2 & P_{+} & \cdots \\
P_{+} & P_{-} & P_{-} & P_{+} & P_{+}-2 & \cdots \\
\hline \cdots & \cdots & \cdots & \cdots & \cdots & \cdots
\end{array}\right) .
$$

We are also interested in the goldstino mass terms and in the mixing terms between goldstinos and gravitinos. Note that the greatest simplification is achieved if we define the 
goldstino basis in a slightly different way than was done for gravitinos in eq. (3.1), namely, by defining

$$
\psi_{5, \rho}^{ \pm}=\frac{\mp \psi_{5, \rho}^{1}+\psi_{5, \rho}^{2}}{\sqrt{2}}, \quad(\rho>0) .
$$

In the $\boldsymbol{\Psi}_{5}=\left(\psi_{5,0}^{2}, \psi_{5,1}^{+}, \psi_{5,1}^{-}, \psi_{5,2}^{+}, \psi_{5,2}^{-}, \ldots\right)^{T}$ basis, we find that the goldstino mass matrix is nothing but the gravitino mass matrix just given above in eq. (3.2). Thus the $4 \mathrm{D}$ lagrangian density suffers a dramatic simplification:

$$
\begin{aligned}
\mathcal{L}_{2 f}^{(4)}= & \frac{1}{2} \epsilon^{m n p q} \overline{\mathbf{\Psi}}_{m}^{T} \bar{\sigma}_{n} \partial_{p} \boldsymbol{\Psi}_{q}-\frac{i}{2} \overline{\mathbf{\Psi}}_{5}^{T} \bar{\sigma}^{m} \partial_{m} \boldsymbol{\Psi}_{5}+\boldsymbol{\Psi}_{m}^{T} \mathcal{M}_{3 / 2} \sigma^{m n} \boldsymbol{\Psi}_{n}+ \\
& +\overline{\mathbf{\Psi}}_{5}^{T} \mathcal{M}_{3 / 2} \boldsymbol{\Psi}_{5}-i \sqrt{\frac{3}{2}} \boldsymbol{\Psi}_{m}^{T} \mathcal{M}_{3 / 2} \sigma^{m} \overline{\mathbf{\Psi}}_{5}+\text { h.c. }
\end{aligned}
$$

Of course, for calculations we are interested in the mass eigenstates, which are obtained as follows. As shown in ref. [4], the eigenvalues $\lambda$ of the matrix $R \mathcal{M}_{3 / 2}$ satisfy the following equation:

$$
\tan (\pi \lambda)=\frac{4 \pi P_{+}}{\pi^{2}\left(P_{-}^{2}-P_{+}^{2}\right)+4}
$$

and therefore the mass eingenvalues are given by

$$
m_{3 / 2}^{a} \equiv m_{3 / 2}^{( \pm \rho)}=\frac{1}{R}\left\{\frac{1}{\pi} \arctan \left[\frac{4 \pi P_{+}}{\pi^{2}\left(P_{-}^{2}-P_{+}^{2}\right)+4}\right] \pm \rho\right\} .
$$

Here $a$ enumerates the components of the vectors $\boldsymbol{\Psi}_{m}$ and $\boldsymbol{\Psi}_{5}$ while $\rho=0,1,2, \ldots$ refers to the KK modes. The orthogonal matrix $Q$ that diagonalizes the gravitino mass matrix

$$
\mathcal{M}_{3 / 2}^{D}=Q^{T} \mathcal{M}_{3 / 2} Q=\operatorname{diag}\left\{m_{3 / 2}^{(0)}, m_{3 / 2}^{(1)}, m_{3 / 2}^{(-1)}, \ldots\right\}
$$

has the following form

$$
Q=\left(\begin{array}{cccccc}
q_{0} & q_{1} & -q_{-1} & q_{2} & -q_{-2} & \cdots \\
\frac{q_{0} c_{0}}{\left(\lambda_{0}-1\right)} & \frac{q_{1} c_{1}}{\left(\lambda_{1}-1\right)} & \frac{-q_{-1} c_{-1}}{\left(\lambda_{-1}-1\right)} & \frac{q_{2} c_{2}}{\left(\lambda_{2}-1\right)} & \frac{-q_{-2} c_{-2}}{\left(\lambda_{-2}-1\right)} & \cdots \\
\frac{q_{0} c_{0}}{\left(\lambda_{0}+1\right)} & \frac{q_{1} c_{1}}{\left(\lambda_{1}+1\right)} & \frac{-q_{-1} c_{-1}}{\left(\lambda_{-1}+1\right)} & \frac{q_{2} c_{2}}{\left(\lambda_{2}+1\right)} & \frac{-q_{-2} c_{-2}}{\left(\lambda_{-2}+1\right)} & \cdots \\
\frac{q_{0} \lambda_{0}}{\left(\lambda_{0}-2\right)} & \frac{q_{1} \lambda_{1}}{\left(\lambda_{1}-2\right)} & \frac{-q_{-1} \lambda_{-1}}{\left(\lambda_{-1}-2\right)} & \frac{q_{2} \lambda_{2}}{\left(\lambda_{2}-2\right)} & \frac{-q_{-2} \lambda_{-2}}{\left(\lambda_{-2}-2\right)} & \cdots \\
\frac{q_{0} \lambda_{0}}{\left(\lambda_{0}+2\right)} & \frac{q_{1} \lambda_{1}}{\left(\lambda_{1}+2\right)} & \frac{-q_{-1} \lambda_{-1}}{\left(\lambda_{-1}+2\right)} & \frac{q_{2} \lambda_{2}}{\left(\lambda_{2}+2\right)} & \frac{-q_{-2} \lambda_{-2}}{\left(\lambda_{-2}+2\right)} & \cdots \\
\ldots & \ldots & \ldots & \ldots & \ldots & \ddots
\end{array}\right),
$$

with

$$
\begin{aligned}
q_{\rho} & =\left[1-2 c_{\rho}^{2}\left(\lambda_{\rho} \frac{\partial \Sigma_{O}}{\partial \lambda_{\rho}}+\Sigma_{O}\right)-2 \lambda_{\rho}^{2}\left(\lambda_{\rho} \frac{\partial \Sigma_{E}}{\partial \lambda_{\rho}}+\Sigma_{E}\right)\right]^{-1 / 2}, \\
c_{\rho} & =\frac{1}{P_{-}}\left[\left(P_{-}^{2}-P_{+}^{2}\right)\left(2 \lambda_{\rho}^{2} \Sigma_{E}+1\right)+P_{+} \lambda_{\rho}\right],
\end{aligned}
$$


where

$$
\Sigma_{E}(\lambda)=\Sigma_{\rho \text { even }} \frac{1}{\lambda^{2}-\rho^{2}}=-\frac{1}{2 \lambda^{2}}+\frac{\pi}{4 \lambda}\left[\frac{1+\cos (\lambda \pi)}{\sin (\lambda \pi)}\right]
$$

and

$$
\Sigma_{O}(\lambda)=\Sigma_{\rho o d d} \frac{1}{\lambda^{2}-\rho^{2}}=-\frac{\pi}{4 \lambda}\left[\frac{1-\cos (\lambda \pi)}{\sin (\lambda \pi)}\right] .
$$

Therefore we introduce the mass eigenvector spinors

$$
\hat{\mathbf{\Psi}}_{m}=\left(\hat{\psi}_{m, 0}^{1}, \hat{\psi}_{m, 1}^{+}, \hat{\psi}_{m, 1}^{-}, \hat{\psi}_{m, 2}^{+}, \hat{\psi}_{m, 2}^{-}, \ldots\right)^{T}
$$

and

$$
\hat{\mathbf{\Psi}}_{5}=\left(\hat{\psi}_{5,0}^{2}, \hat{\psi}_{5,1}^{+}, \hat{\psi}_{5,1}^{-}, \hat{\psi}_{5,2}^{+}, \hat{\psi}_{5,2}^{-}, \ldots\right)^{T}
$$

related to $\boldsymbol{\Psi}_{m}$ and $\boldsymbol{\Psi}_{5}$ by

$$
\hat{\mathbf{\Psi}}_{m}=Q^{T} \boldsymbol{\Psi}_{m}, \quad \hat{\boldsymbol{\Psi}}_{5}=Q^{T} \boldsymbol{\Psi}_{5},
$$

in terms of which the lagrangian given in eq. (3.4) can be re-expressed as

$$
\begin{aligned}
\mathcal{L}_{2 f}^{(4)}= & \frac{1}{2} \epsilon^{m n p q} \hat{\mathbf{\Psi}}_{m}^{T} \bar{\sigma}_{n} \partial_{p} \hat{\mathbf{\Psi}}_{q}-\frac{i}{2} \overline{\hat{\mathbf{\Psi}}}_{5}^{T} \bar{\sigma}^{m} \partial_{m} \hat{\mathbf{\Psi}}_{5}+\hat{\mathbf{\Psi}}_{m}^{T} \mathcal{M}_{3 / 2}^{D} \sigma^{m n} \hat{\mathbf{\Psi}}_{n}+ \\
& +\overline{\hat{\mathbf{\Psi}}}_{5}^{T} \mathcal{M}_{3 / 2}^{D} \hat{\mathbf{\Psi}}_{5}-i \sqrt{\frac{3}{2}} \hat{\mathbf{\Psi}}_{m}^{T} \mathcal{M}_{3 / 2}^{D} \sigma^{m} \overline{\hat{\mathbf{\Psi}}}_{5}+\text { h.c. }
\end{aligned}
$$

In our discussion we have assumed $P_{0} \neq P_{\pi}$; when $P_{0}=-P_{\pi}$ the transformation in eq. (3.8) is singular. In that case a $N=1$ SUSY is left unbroken [1] and a field redefinition to get the fermion bilinear lagrangian in a diagonalized form has been identified [3].

Once we have identified the mass and mixing terms of the goldstino and gravitino KK tower, we turn to the proof of the ET.

\section{The Equivalence Theorem}

The Equivalence Theorem we will prove here is an extension of the corresponding theorem in spontaneously broken supergravity in four dimensions, first suggested in [13] and proved in [5]: at center of mass energies $E \gg m_{3 / 2}$, the S-matrix elements with external helicity $\pm 1 / 2$ gravitinos (longitudinal gravitinos) are equivalent to the S-matrix elements with corresponding goldstinos. In order to use the known results it is convenient to rewrite the lagrangian density in eq. (3.14) using Majorana spinors defined in terms of the corresponding Weyl ones as

$$
\psi^{(M)}=\left(\frac{\psi_{\alpha}}{\bar{\psi}^{\dot{\alpha}}}\right), \quad \bar{\psi}^{(M)}=\left(\psi^{\alpha}, \bar{\psi}_{\dot{\alpha}}\right) .
$$

The result is

$$
\begin{aligned}
\mathcal{L}_{2 f}^{(4)}= & \frac{1}{2} \epsilon^{m n p q} \hat{\mathbf{\Psi}}_{m}^{(M) T} \gamma_{5} \gamma_{n} \partial_{p} \hat{\mathbf{\Psi}}_{q}^{(M)}-\frac{i}{2} \overline{\mathbf{\Psi}}_{5}^{(M) T} \gamma^{m} \partial_{m} \hat{\mathbf{\Psi}}_{5}^{(M)}+\hat{\mathbf{\Psi}}_{m}^{(M) T} \mathcal{M}_{3 / 2}^{D} \gamma^{m n} \hat{\mathbf{\Psi}}_{n}^{(M)}+ \\
& +\overline{\mathbf{\Psi}}_{5}^{(M) T} \mathcal{M}_{3 / 2}^{D} \hat{\mathbf{\Psi}}_{5}^{(M)}-i \sqrt{\frac{3}{2}} \hat{\mathbf{\Psi}}_{m}^{(M) T} \mathcal{M}_{3 / 2}^{D} \gamma^{m} \hat{\mathbf{\Psi}}_{5}^{(M)}
\end{aligned}
$$


with $\gamma^{m n}$ given in appendix A. Apart from the mass, $\boldsymbol{\Psi}_{5}$ and metric sign conventions, we find that $\mathcal{L}_{2 f}^{(4)}$ is nothing but a sum of infinite copies of the lagrangian (2.6) in ref. [5, one for each component of the gravitino and goldstino vectors. This lagrangian was the starting point for the proof of the ET between $\pm 1 / 2$ helicity gravitinos and goldstinos.

\section{1 $R_{\xi}$ gauge fixing}

The basic idea for the proof was to use the analogous of $R_{\xi}$ gauges in the non abelian gauge theories. Contrary to the unitary gauges used in [3, 1 , within these so called renormalizable or t'Hooft-Veltman gauges, the gravitinos and the goldstinos coexist in the lagrangian, and is possible to relate them at high energy through the ET. We recall that the main virtue of these generalized $R_{\xi}$ gauges is to cancel the mixing term between the gravitino and the goldstino fields. This is achieved by adding to the lagrangian density a gauge fixing term. It is easy to check that, in the present case, the gauge fixing term has the following form:

$$
\mathcal{L}_{G F}^{(4)}=-\frac{i}{2} \xi \sum_{a} \overline{\hat{F}_{a}} \not \partial \hat{F}_{a}
$$

with

$$
\hat{F}_{a}=\gamma^{m} \hat{\Psi}_{m, a}^{(M)}-\sqrt{\frac{3}{2}} m_{3 / 2}^{a} \frac{1}{\xi \not \partial} \hat{\Psi}_{5, a}^{(M)} .
$$

It is interesting to rewrite the gauge fixing term in a $5 \mathrm{D}$ form. In fact, since we have a copy of the lagrangian for each component of the vectors $\hat{\mathbf{\Psi}}_{m}=\left(\hat{\psi}_{m, 0}^{1}, \hat{\psi}_{m, 1}^{+}, \hat{\psi}_{m, 1}^{-}, \hat{\psi}_{m, 2}^{+}, \hat{\psi}_{m, 2}^{-}, \ldots\right)^{T}$ and $\hat{\mathbf{\Psi}}_{5}=\left(\hat{\psi}_{5,0}^{2}, \hat{\psi}_{5,1}^{+}, \hat{\psi}_{5,1}^{-}, \hat{\psi}_{5,2}^{+}, \hat{\psi}_{5,2}^{-}, \ldots\right)^{T}$ (see eq. (3.14) ), we need a gauge fixing term for each component, each one relating a gravitino KK state with its corresponding KK goldstino mode. As we have already remarked, these gauge fixing terms are found by requiring the cancellation of the gravitino-goldstino mixing term in eq. (4.2). The 4D gauge fixing lagrangian can be written:

$$
\mathcal{L}_{G F}^{(4)}=-\frac{i}{2} \xi \overline{\hat{\mathbf{F}}}^{T} \not \partial \hat{\mathbf{F}} ; \quad \hat{\mathbf{F}}=\gamma^{m} \hat{\mathbf{\Psi}}_{m}^{(M)}-\sqrt{\frac{3}{2}} \mathcal{M}_{3 / 2}^{D} \frac{1}{\xi \not \partial} \hat{\mathbf{\Psi}}_{5}^{(M)}
$$

We show in appendix $\mathrm{B}$ how to generate the $4 \mathrm{D}$ gauge fixing term in eq. (4.3) from the original $5 \mathrm{D}$ lagrangian in eq. (2.6). We check there that the $5 \mathrm{D}$ gauge fixing term can be written as

$$
\mathcal{L}_{G F}^{(5)}=-\frac{i}{2} \xi \bar{H} \Gamma^{m} \partial_{m} H=-\frac{i}{4} \xi\left[\overline{H^{1}} \bar{\sigma}^{m} \partial_{m} H^{1}+\overline{H^{2}} \bar{\sigma}^{m} \partial_{m} H^{2}\right]+\text { h.c. }
$$

where $\Gamma^{m}$ is given in appendix A, $H$ is a generalized Majorana 5D spinor: $H=\left(H_{\alpha}^{1},{\overline{H^{2}}}^{\dot{\alpha}}\right)$ and $\bar{H}=\left(H^{2 \alpha}, \bar{H}_{\dot{\alpha}}\right)$, with

$$
\begin{aligned}
& H^{1}=\sigma^{m}{\overline{\psi^{1}}}_{m}-\sqrt{\frac{3}{2}} \frac{1}{\xi \partial^{2}} \partial_{m} \sigma^{m}\left(\partial_{5}{\overline{\psi^{1}}}_{5}-\kappa^{3}\left[\delta\left(x^{5}\right) P_{0}+\delta\left(x^{5}-\pi \kappa\right) P_{\pi}\right]{\overline{\psi^{2}}}_{5}\right), \\
& H^{2}=\sigma^{m}{\overline{\psi^{2}}}_{m}-\sqrt{\frac{3}{2}} \frac{1}{\xi \partial^{2}} \partial_{m} \sigma^{m} \partial_{5}{\overline{\psi^{2}}}_{5} .
\end{aligned}
$$


We have checked that the gauge fixing term needed in $5 \mathrm{D}$ is precisely the one that cancels the $5 \mathrm{D}$ mixing terms in eq. (2.6). Note that it is made of two gauge fixing functions, since we have two gravitinos, exactly as it happens when we have several gauge fields and we have to gauge fix each one of them. Also, note that the gauge fixing term breaks the 5D Lorentz invariance: first because the mixings induced by the branes break it by the $\delta$-terms, and second because of the presence of $\partial^{m}$ derivatives. This is also what happens, for instance, in electrodynamics when using Coulomb gauges, which are originally thought to deal with static problems, where three coordinates are enough to describe an otherwise four dimensional theory.

\subsection{The Equivalence Theorem for KK modes}

The sum in eq. (4.3) is over the components of the vectors $\hat{\mathbf{\Psi}}_{m}^{(M)}$ and $\hat{\mathbf{\Psi}}_{5}^{(M)}$ and $m_{3 / 2}^{a}$ are the eigenvalues of the gravitino mass matrix. From the path integral point of view, a gauge fixing as in eq. (4.3) is a delta functional $\delta(\not \partial F)$, which intuitively corresponds to imposing $\not \partial F=0$ throughout the calculations. That is, we get a relation between $\not \partial \gamma^{m} \hat{\Psi}_{m, a}^{(M)}$ and $\hat{\Psi}_{5, a}^{(M)}$ for each component $a$. This is precisely what we need to relate the on-shell amplitudes of helicity $\pm 1 / 2$ gravitinos and their KK excitations with the corresponding amplitudes for goldstinos.

The rigorous proof [5 makes use of the BRS invariance to get a set of relevant Ward identities leading to the corresponding relations between S-matrix elements for external gravitinos and goldstinos. The main steps are the following. In the high energy regime, that is, for energies much larger than the gravitino mass $\left(s \gg m_{3 / 2}^{2}\right)$, one can approximate the wave function corresponding to the longitudinal components of the gravitino as

$$
\psi^{ \pm 1 / 2}=-\bar{E}_{m}^{ \pm 1 / 2} \psi^{m}
$$

where

$$
E_{m}^{ \pm 1 / 2}(p) \sim\left(i \sqrt{\frac{2}{3}} \frac{p_{m}}{m_{3 / 2}}+\mathcal{O}\left(\frac{m_{3 / 2}}{p}\right)\right) u^{ \pm 1 / 2}(p)
$$

with $u^{ \pm 1 / 2}(p)$ a Dirac spinor with appropriate polarization. Using the BRS invariance and the relation between the goldstino and the gravitino wave operators one obtains

$$
S\left(A, \psi^{ \pm 1 / 2}, B\right)=S\left(A, \psi_{5}, B\right)+\mathcal{O}\left(\frac{m_{3 / 2}}{\sqrt{s}}\right)
$$

where $S\left(A, \psi^{ \pm 1 / 2}\left(\psi_{5}\right), B\right)$ denotes the $S$ matrix elements for the longitudinal gravitino (goldstino) and any other $A$ and $B$ physical states.

We can rephrase all the proof in terms of the components of the vectors $\hat{\mathbf{\Psi}}_{m}^{(M)}$ and $\hat{\mathbf{\Psi}}_{5}^{(M)}$, and formulate an Equivalence Theorem relating the $S$ matrix elements involving the longitudinal components of KK gravitinos with the ones involving the corresponding KK goldstinos. At energies where we can neglect the masses of the gravitinos involved in the scattering amplitude under consideration, it reads:

$$
T\left(\hat{\Psi}_{a}^{ \pm 1 / 2}, \hat{\Psi}_{b}^{ \pm 1 / 2}, \ldots \hat{\Psi}_{c}^{ \pm 1 / 2}\right)=T\left(\hat{\Psi}_{5 a}^{(M)}, \hat{\Psi}_{5 b}^{(M)}, \ldots \hat{\Psi}_{5 c}^{(M)}\right)+O\left(\max \left[\frac{\left|m_{3 / 2}^{a}\right|}{\sqrt{s}}, \frac{\left|m_{3 / 2}^{b}\right|}{\sqrt{s}}, \ldots\right]\right)
$$


where $\hat{\Psi}_{a}^{ \pm 1 / 2}$ denotes the $a$ component of the vector $\hat{\boldsymbol{\Psi}}^{ \pm 1 / 2}=-\bar{E}_{m}^{ \pm 1 / 2} \hat{\boldsymbol{\Psi}}^{(M) m}$ and $\hat{\Psi}_{5 a}^{(M)}$ denotes the $a$ component of the vector $\hat{\Psi}_{5}^{(M)}$. Note that the ET holds for the mass eigenfields and for energies higher than the mass of the heaviest KK mode in the amplitude. Finally, let us remark that the ET is most useful in the Landau gauge, $\xi \rightarrow \infty$, where the gauge dependent complicated goldstino kinetic terms coming from the gauge fixing function cancel, and we just have to deal with the usual simple propagators for spin $1 / 2$ fields.

\subsection{The $R \rightarrow 0$ limit}

It is instructive to obtain the values of the $Q$ elements in the $R \sim \kappa^{1 / 3} \rightarrow 0$ limit, $(R$ and $\kappa$ are related by requiring a finite $4 \mathrm{D}$ Planck mass $M_{4}$ ). In such case the physics is basically four dimensional with a very tiny fifth dimension, which is a natural limit in the brane induced supersymmetry breaking scenarios. Indeed the scale associated to the orbifold $(\pi \kappa)^{-1}$ comes out to be of the order $10^{15} \mathrm{GeV}$, while the scale of the supersymmetry breaking $\left[\left(P_{0}+P_{\pi}\right) / 2\right]^{1 / 3}$ is of the order $10^{13} \mathrm{GeV}$ (see for instance [14] and references therein). In this case $P_{+} \ll 1$ and the lightest gravitino mass turns out to be

$$
m_{3 / 2}^{(0)} \sim \frac{P_{+}}{R} \sim \frac{1}{2} \frac{P_{0}+P_{\pi}}{M_{4}^{2}} \sim 200 \mathrm{GeV} .
$$

In this limit, the matrix $Q$ reduces to:

$$
Q=\left(\begin{array}{cccc}
1+O\left(\kappa^{6}\right) & O\left(\kappa^{3}\right) & O\left(\kappa^{3}\right) & \cdots \\
O\left(\kappa^{3}\right) & 1+O\left(\kappa^{6}\right) & O\left(\kappa^{3}\right) & \cdots \\
O\left(\kappa^{3}\right) & O\left(\kappa^{3}\right) & 1+O\left(\kappa^{6}\right) & \cdots \\
\ldots & \ldots & \ldots & \ddots
\end{array}\right)
$$

That is, the mass eigenfields are basically those initially in the lagrangian except for an $O\left(\kappa^{3}\right)$ correction. Consequently,

$$
T\left(\hat{\Psi}_{a}^{ \pm 1 / 2}, \hat{\Psi}_{b}^{ \pm 1 / 2}, \ldots \hat{\Psi}_{c}^{ \pm 1 / 2}\right) \simeq T\left(\Psi_{5 a}^{(M)}, \Psi_{5 b}^{(M)}, \ldots \Psi_{5 c}^{(M)}\right) .
$$

In other words, in this limit we can calculate scattering amplitudes involving helicity $\pm 1 / 2$ gravitinos in a simpler way by using directly the vertices of the interaction lagrangian for goldstinos, without the need for a rotation. Note that terms we are neglecting, with respect to the complete formulation of the ET in eq. (4.12) are suppressed by $O\left(\kappa^{3}\right)$ factors (to obtain the precise order in $\kappa$ we would need to know the superpotential). Of course, to get higher order corrections in $\kappa$ we need the complete formulation.

\section{Conclusions}

We have presented a proof of the Equivalence Theorem for massive Kaluza Klein gravitino modes in 5D supergravity with brane induced supersymmetry breaking. This theorem holds 
for energies higher than the mass of any of the gravitinos involved, and allows to perform calculations of amplitudes containing on-shell helicity $\pm 1 / 2$ gravitinos by substituting them with their corresponding goldstino fields.

In particular, we have identified the four and five dimensional gauge fixing functions from which the ET follows. We have also identified the goldstino field combinations that correspond to each gravitino Kaluza Klein mode, providing the expressions of the rotation matrices to obtain these eigenfields in the general case. Finally, we have studied the behavior of these transformations in the limit of a small extra dimension.

The results presented here can be useful in further studies 10 of observables involving massive Kaluza Klein gravitinos, for instance in a cosmological context, that could provide bounds on the parameters of these supersymmetry breaking models.

\section{Acknowledgments}

The authors thank A.L. Maroto for his comments and careful reading of the manuscript, as well as partial support from the INFN-CICYT Florence-Madrid Collaboration. J.R.P. thanks the hospitality of the INFN Sezione di Firenze as well as partial support from the Spanish CICYT projects PB98-0782 and BFM2000 1326.

\section{A. Notation}

We follow the conventions of Wess and Bagger, [16], in particular we recall that

$$
\begin{aligned}
\eta_{m n} & =(-1,1,1,1), \quad \Gamma^{5}=\left(\begin{array}{cc}
-i & 0 \\
0 & i
\end{array}\right)=i \gamma^{5}, \quad \Gamma^{m}=\left(\begin{array}{cc}
0 & \sigma^{m} \\
\bar{\sigma}^{m} & 0
\end{array}\right), \\
\epsilon_{0123} & =-1, \quad \sigma^{m}=(-I, \vec{\sigma}), \quad \bar{\sigma}^{m}=(-I,-\vec{\sigma}), \\
\sigma^{m n} & =\frac{1}{4}\left(\sigma^{m} \bar{\sigma}^{n}-\sigma^{n} \bar{\sigma}^{m}\right), \quad \bar{\sigma}^{m n}=\frac{1}{4}\left(\bar{\sigma}^{m} \sigma^{n}-\bar{\sigma}^{n} \sigma^{m}\right), \\
\gamma^{m n} & =\left(\begin{array}{cc}
\sigma^{m n} & 0 \\
0 & \bar{\sigma}^{m n}
\end{array}\right) .
\end{aligned}
$$

\section{B. Gauge fixing term in 5D supergravity}

Let us show how to generate the $4 \mathrm{D}$ gauge fixing term in eq. (4.5) from the original 5D lagrangian in eq. (2.6). First, we rewrite it in terms of Weyl spinors. For that purpose it is convenient to note that

$$
\overline{\hat{\mathbf{F}}}^{T} \not \partial \hat{\mathbf{F}}=\left(\overline{-\gamma_{5} \hat{\mathbf{F}}}\right)^{T} \not \partial\left(-\gamma_{5} \hat{\mathbf{F}}\right)
$$

where $-\gamma_{5} \hat{\mathbf{F}}$ is a Majorana spinor (whereas $\hat{\mathbf{F}}$ is not). Then we can simply re-express the $4 \mathrm{D}$ gauge fixing lagrangian in terms of one Weyl spinor $\hat{\mathbf{f}}$ as follows:

$$
\begin{aligned}
-\gamma_{5} \hat{\mathbf{F}} & =\left(\begin{array}{l}
\hat{\mathbf{f}}_{\alpha} \\
\hat{\hat{\mathbf{f}}}^{\dot{\alpha}}
\end{array}\right), \\
\hat{\mathbf{f}} & =\sigma^{m} \overline{\hat{\mathbf{\Psi}}}_{m}-\sqrt{\frac{3}{2}} \mathcal{M}_{3 / 2}^{D} \frac{1}{\xi \partial^{2}} \partial_{m} \sigma^{m} \overline{\hat{\mathbf{\Psi}}}_{5}, \\
\mathcal{L}_{G F}^{(4)} & =-\frac{i}{2} \xi \overline{\hat{\mathbf{f}}}^{T} \bar{\sigma}^{m} \partial_{m} \hat{\mathbf{f}}+\text { h.c. }
\end{aligned}
$$


Let us now recall that the transformations $\hat{\mathbf{\Psi}}_{m}=Q^{T} \boldsymbol{\Psi}_{m}, \hat{\mathbf{\Psi}}_{5}=Q^{T} \boldsymbol{\Psi}_{5}$ are orthogonal, so that the above gauge fixing term can be recast by replacing $\hat{\mathbf{\Psi}}_{m} \rightarrow \boldsymbol{\Psi}_{m}, \hat{\mathbf{\Psi}}_{5} \rightarrow \boldsymbol{\Psi}_{5}$ and $\mathcal{M}_{3 / 2}^{D} \rightarrow \mathcal{M}_{3 / 2}=Q \mathcal{M}_{3 / 2}^{D} Q^{T}$. Recalling that the $\psi^{ \pm}$fields are related to the $\psi^{1,2}$ fields by eqs. (3.1), (3.3), we can write

$$
\boldsymbol{\Phi}_{\mathbf{m}}=\left(\begin{array}{c}
\psi_{m, 0}^{1} \\
\psi_{m, 1}^{1} \\
\psi_{m, 1}^{2} \\
\psi_{m, 2}^{1} \\
\psi_{m, 2}^{2} \\
\vdots
\end{array}\right)=\Omega \boldsymbol{\Psi}_{\mathbf{m}} \equiv\left(\begin{array}{cccccc}
1 & & & & & \\
& \frac{1}{\sqrt{2}} & \frac{1}{\sqrt{2}} & & & \\
& \frac{1}{\sqrt{2}} & \frac{-1}{\sqrt{2}} & & & \\
& & & \frac{1}{\sqrt{2}} & \frac{1}{\sqrt{2}} & \\
& & \frac{1}{\sqrt{2}} & \frac{-1}{\sqrt{2}} & \\
& & & & & \ddots
\end{array}\right)\left(\begin{array}{c}
\psi_{m, 0}^{1} \\
\psi_{m, 1}^{+} \\
\psi_{m, 1}^{-} \\
\psi_{m, 2}^{+} \\
\psi_{m, 2}^{-} \\
\vdots
\end{array}\right)
$$

and

$$
\boldsymbol{\Phi}_{5}=\left(\begin{array}{c}
\psi_{5,0}^{2} \\
\psi_{5,1}^{1} \\
\psi_{5,1}^{2} \\
\psi_{5,2}^{1} \\
\psi_{5,2}^{2} \\
\vdots
\end{array}\right)=\Omega_{5} \boldsymbol{\Psi}_{5} \equiv\left(\begin{array}{cccccc}
1 & & & & & \\
& \frac{-1}{\sqrt{2}} & \frac{1}{\sqrt{2}} & & & \\
& \frac{1}{\sqrt{2}} & \frac{1}{\sqrt{2}} & & & \\
& & & \frac{-1}{\sqrt{2}} & \frac{1}{\sqrt{2}} & \\
& & \frac{1}{\sqrt{2}} & \frac{1}{\sqrt{2}} & \\
& & & & & \ddots
\end{array}\right)\left(\begin{array}{c}
\psi_{5,0}^{2} \\
\psi_{5,1}^{+} \\
\psi_{5,1}^{-} \\
\psi_{5,2}^{+} \\
\psi_{5,2}^{-} \\
\vdots
\end{array}\right)
$$

Since $\Omega=\Omega^{T}=\Omega^{-1}, \Omega_{5}=\Omega_{5}^{T}=\Omega_{5}^{-1}$, again we can simply rewrite the gauge fixing term as

$$
\mathcal{L}_{G F}^{(4)}=\frac{-i}{2} \xi \overline{\mathbf{h}}^{T} \bar{\sigma}^{m} \partial_{m} \mathbf{h}+\text { h.c. } ; \quad \mathbf{h}=\sigma^{m} \overline{\mathbf{\Phi}}_{m}-\sqrt{\frac{3}{2}} \frac{1}{\xi \partial^{2}} \partial_{m} \sigma^{m} \Omega \mathcal{M}_{3 / 2} \Omega_{5} \overline{\mathbf{\Phi}}_{5} .
$$

Let us write explicitly

$$
\Omega \mathcal{M}_{3 / 2} \Omega_{5} \overline{\boldsymbol{\Phi}}_{5}=\left(\begin{array}{c}
P_{+} \bar{\psi}_{5,0}^{2}+\sqrt{2} \sum_{\rho=1}^{\infty}\left(P_{0}+(-1)^{\rho} P_{\pi}\right) \bar{\psi}_{5, \rho}^{2} \\
\sqrt{2}\left[P_{-} \bar{\psi}_{5,0}^{2}+\sqrt{2} \sum_{\rho=1}^{\infty}\left(P_{0}+(-1)^{\rho+1} P_{\pi}\right) \bar{\psi}_{5, \rho}^{2}\right] \\
0 \\
\sqrt{2}\left[P_{+} \bar{\psi}_{5,0}^{2}+\sqrt{2} \sum_{\rho=1}^{\infty}\left(P_{0}+(-1)^{\rho+2} P_{\pi}\right) \bar{\psi}_{5, \rho}^{2}\right] \\
0 \\
\vdots
\end{array}\right)+\left(\begin{array}{c}
0 \\
-\bar{\psi}_{5,1}^{1} \\
\bar{\psi}_{5,1}^{2} \\
-2 \bar{\psi}_{5,2}^{1} \\
2 \bar{\psi}_{5,2}^{2} \\
\vdots
\end{array}\right) .
$$

The first vector is related to the physics on the branes and the second to the bulk. It is straightforward to check that the gauge fixing term in eq. (B.7) comes from a $5 \mathrm{D}$ gauge fixing term:

$$
\mathcal{L}_{G F}^{(5)}=-\frac{i}{2} \xi \bar{H} \Gamma^{m} \partial_{m} H=-\frac{i}{4} \xi\left[\overline{H^{1}} \bar{\sigma}^{m} \partial_{m} H^{1}+\overline{H^{2}} \bar{\sigma}^{m} \partial_{m} H^{2}\right]+\text { h.c. } ;
$$

where $\Gamma^{m}$ is given in appendix A, $H$ is a generalized Majorana 5D spinor: $H=\left(H_{\alpha}^{1},{\overline{H^{2}}}^{\dot{\alpha}}\right)$ and $\bar{H}=\left(H^{2 \alpha}, \bar{H}_{\dot{\alpha}}\right)$, with

$$
\begin{aligned}
H^{1} & =\sigma^{m}{\overline{\psi^{1}}}_{m}-\sqrt{\frac{3}{2}} \frac{1}{\xi \partial^{2}} \partial_{m} \sigma^{m}\left(\partial_{5}{\overline{\psi^{1}}}_{5}-\kappa^{3}\left[\delta\left(x^{5}\right) P_{0}+\delta\left(x^{5}-\pi \kappa\right) P_{\pi}\right]{\overline{\psi^{2}}}_{5}\right), \\
H^{2} & =\sigma^{m}{\overline{\psi^{2}}}_{m}-\sqrt{\frac{3}{2}} \frac{1}{\xi \partial^{2}} \partial_{m} \sigma^{m} \partial_{5} \bar{\psi}_{5}{ }_{5} .
\end{aligned}
$$


It is easy to verify that this term exactly cancels the mixing terms between the goldstinos and the gravitinos in the 5D lagrangian density given in eq. (2.6).

\section{References}

[1] P. Hořava and E. Witten, Eleven-dimensional supergravity on a manifold with boundary, Nucl. Phys. B 475 (1996) 94 [hep-th/9603142; Heterotic and type-I string dynamics from eleven dimensions, Nucl. Phys. B 460 (1996) 506 hep-th/9510209.

[2] P. Hořava, Gluino condensation in strongly coupled heterotic string theory, Phys. Rev. D 54 (1996) 7561 hep-th/9608019.

[3] K.A. Meissner, H.P. Nilles and M. Olechowski, Supersymmetry breakdown at distant branes: the super-Higgs mechanism, Nucl. Phys. B 561 (1999) 30 hep-th/9905139.

[4] J. Bagger, F. Feruglio and F. Zwirner, Brane induced supersymmetry breaking, J. High Energy Phys. 02 (2002) 010 hep-th/0108010.

[5] R. Casalbuoni, S. De Curtis, D. Dominici, F. Feruglio and R. Gatto, High-energy equivalence theorem in spontaneously broken supergravity, Phys. Rev. D 39 (1989) 2281; A gravitino goldstino high-energy equivalence theorem, Phys. Lett. B 215 (1988) 313.

[6] R. Kallosh, L. Kofman, A.D. Linde and A. Van Proeyen, Gravitino production after inflation, Phys. Rev. D 61 (2000) 103503 hep-th/9907124;

G.F. Giudice, A. Riotto and I. Tkachev, Thermal and non-thermal production of gravitinos in the early universe, J. High Energy Phys. 11 (1999) 036 hep-ph/9911302;

A.L. Maroto and J.R. Pelaez, The equivalence theorem and the production of gravitinos after inflation, Phys. Rev. D 62 (2000) 023518 hep-ph/9912212.

[7] A. Mazumdar, Interesting consequences of brane cosmology, Phys. Rev. D 64 (2001) 027304 hep-ph/0007269; Post-inflationary brane cosmology, Nucl. Phys. B 597 (2001) 561 hep-ph/0008087.

[8] T. Bhattacharya and P. Roy, Tree unitarity in broken supergravity, 1. Single gravitino amplitude, Nucl. Phys. B 328 (1989) 469; Tree unitarity in broken supergravity, 2. Double gravitino amplitude, Nucl. Phys. B 328 (1989) 481.

[9] S. De Curtis, D. Dominici and J.R. Pelaez, Strong tree level unitarity violations in the extra dimensional standard model with scalars in the bulk, Phys. Rev. D 67 (2003) 076010 hep-ph/0301059.

[10] S. De Curtis, D. Dominici and J.R. Pelaez, The equivalence theorem for gauge boson scattering in a five dimensional standard model, Phys. Lett. B 554 (2003) 164 hep-ph/0211353.

[11] E. Cremmer, Supergravities in 5 dimensions, in Superspace and supergravity, S.W. Hawking and M. Rocek eds., Cambridge University Press, Cambridge 1981, pp. 267-282;

A.H. Chamseddine and H. Nicolai, Coupling the $\mathrm{SO}(2)$ supergravity through dimensional reduction, Phys. Lett. B 96 (1980) 89.

[12] L. Dolan, Symmetries of massive fields in Kaluza-Klein supergravity, Phys. Rev. D 30 (1984) 2474; The mass spectrum of supergravity on the circle, in Proc. of 13th Int. Colloq. on Group Theoretical Methods in Physics, W.W. Zachary ed., World Scientific, Singapore 1984. 
[13] P. Fayet, Mixing between gravitational and weak interactions through the massive gravitino, Phys. Lett. B 70 (1977) 461.

[14] H.P. Nilles, Hidden sector supergravity breakdown, Nucl. Phys. 101 (Proc. Suppl.) (2001) 237 hep-ph/0106063.

[15] A.L. Maroto and J.R. Pelaez, in preparation.

[16] J. Wess and J. Bagger, Supersymmetry and Supergravity, Univesity Press, Princeton 1992. 